\section{THE PROLAPSE SYNDROME*}

BY

A. C. PALMER, F.R.C.S.

GYNAECOLOGIST AND OBSTETRICIAN, KING'S COLLEGE HOSPITAL

Briefly, the term " prolapse syndrome" means prolapse associated with excessive bleeding from the cavity of the uterus. Different women present differing forms and degrees of prolapse, but the common form consists of a cystocele and rectocele, with deficient perineum, and more or less descent of the cervix, accompanied by a hernial protrusion of the pouch of Douglas. When on straining the cervix protrudes for an inch or more from the vulva, the form of the prolapse becomes typical of that denoted by the term.

In the early part of this century removal of the uterus by the vaginal route was almost wholly abandoned in favour of the abdominal route. This change was partly due to the fact that gynaecologists were becoming surgeons rather than physicians, and partly because in a large number of cases abdominal hysterectomy is obviously the method of choice.

In 1919 I saw vaginal hysterectomy performed a few times with mild surprise. During the next few years it was less rare, and $I$ saw it often enough to learn a technique and suspect that there might be occasions when it had some advantage over the abdominal method: advantage from the point of view of its being less exhausting to both patient and surgeon. To the eye of one accustomed to the abdominal approach vaginal hysterectomy seemed a kind of fiddling, delving in the dark, affair-a cobbling up of tissues with little resemblance to ordered surgical procedure. In the course of experience this impression became modified, and one was surprised to find that the steps of the technique naturally followed each other in a simple and orderly manner.

During this period it became my portion to operate on a number of 14-stone patients possessed of an enlarged uterus, the " adipose sporran," $\dagger$ varying degrees of prolapse, and complaining of excessive bleeding, accompanied by such general impairment of physical capacity as merited hysterectomy for its cure. These cases were dealt with by the abdominal route, the three or more inches of subcutaneous fat and the "sporran" making the opening and closing of the abdominal wall, in itself, an operation of some difficulty. The subperitoneal fat and the heavily laden omentum further narrowed the approach to the uterus, partially obscuring landmarks and impeding the satisfactory application of ligatures. The majority of the patients sustained a fair amount of shock and passed through a stormy post-operative period. In some, mild suppuration occurred in the subcutaneous fat, wounds broke down, to heal finally by granulation ; a few left the hospital with incipient incisional herniae, while two patients, including one of mine, burst their abdominal wounds about the twelfth day after operation, depositing the intestines in the bed. These were resutured immediately, and in aue course recovered. There was great difficulty in finding anything in the way of separate layers for suture, the cut surface on each side being fused into a solid mass.

Being now free from bleeding, these patients, in the course of time, regained some of their former energy and physical enterprise, and began to notice their disability from prolapse. Some reappeared complaining of dragging pain, of something coming down giving difficulty

\footnotetext{
* Read in the Section of Obstetrics and Gynaccology at the Annual Meeting of the British Medical Association, Bournemouth, 1934

$t$ Adipose sporran: A fold of abdominal skin and subcutaneous
} fat which rolls down over the symphysis pubis. in walking, or of stress incontinence of urine, and asked for relief. They required anterior colporrhaphy and posterior colpoperineorrhaphy, or, more briefly, reconstruction of the pelvic diaphragm, and in the case of some, whose former operation had been a subtotal hysterectomy, a hypertrophied actively discharging cervix required removal as well. A few required belts or further operation for their incisional herniae.

Remembering that these patients had already spent an hour on the operating table, experienced a stormy post-operative period and been three weeks or more in hospital, it seemed unsatisfactory that they should now reappear demanding another operation for part of their original complaint, and in a few cases further surgical treatment as a result of the former abdominal incision. Of course one knows that incisional herniae are sometimes unavoidable; but they are particularly liable to occur in the fat abdomen. This question of herniae, while being of interest, is secondary to the main considerationthat of a second operation for the treatment of part of the original complaint. The reconstruction operation being done, the patients spent on the average another three weeks in hospital, and returned to their vocations after a further period of two or three weeks' convalescence.

\section{A Combined Operation}

In thinking about this matter the question naturally arises: "Why not do a vaginal hysterectomy and combine it with the reconstruction operation?" There did not seem to be any very obvious objection, except the general consensus of opinion that vaginal hysterectomy formed no part of the operative treatment of prolapse, and was in fact contraindicated on the ground that in removal of the cervix one lost a stable suture point for the anterior vaginal mucosa. This reason I personally was unable to appreciate, because in order to render the cervix stable it had first to be hitched up in the pelvis by shortening the lateral ligaments, and further, in many cases, owing to hypertrophy and discharge, the greater portion of the cervix had to be removed as part of the reconstruction operation. The presence of the infected cervix might offer a further objection to opening the abdomen from below, or to the making of free vaginal incisions in its neighbourhood, and for some time this consideration made one hesitate to combine the two operations. On the other hand, amputation of the cervix is combined with repair operations, and here the infected cervix is actually incised, whereas in hysterectomy the incisions are not made in the cervix, but in relatively healthy adjacent tissue, even further away from the actual cervix than in the abdominal route; moreover, the condition of the cervix can be improved before operation by suitable douching.

With these considerations in view I began tentatively to combine the two operations, and up to the present time have found the procedure satisfactory to the patient and surgeon in suitably selected cases-namely, those whose symptoms and physical signs are denoted by the term " prolapse syndrome." This term, while including the prolapse sign and symptom complex, is meant to have a wider application, and extend to those patients who have an enlarged or retroverted uterus, or both, and complain of excessive bleeding, the condition of the uterus being such as to render practicable its removal from below.

\section{Common Prolapse Symptoms}

1. Girdle-ache, including sacral backache and dragging pain in the inguinal regions.

2. A sense of discomfort from something coming down in the perineal region. 
3. Frequency of micturition, varying in degree up to stress incontinence.

4. Irritation from excessive cervical discharge.

5. Undue fatigue at the end of a routine day.

Perhaps the outstanding feature is a sense of diminished physical capacity; the patient can now undertake only with difficulty and resultant fatigue what she formerly accomplished with ease. The ordinary daily round of household and social duties produces exhaustion. This state of affairs is due partly to anaemia and partly to the discomfort and other disturbances arising from prolapse.

These symptoms combine to reduce the patients' physical capacity, and make them unwilling to undertake the discomfort of walking or other forms of activity. Other difficulties arise in the later stages of prolapse, but these need not concern us here. A number of multiparne-it is not possib'e to give percentages-with prolapse have, in addition to their hypertrophied cervix, an enlarged uterus, which may be retroverted. The enlargement of the uterus may be due to a chronic subinvolution, to multiple small fibroids, rarely to diffuse adenomyoma, or even carcinoma of the body; these conditions give rise to excessive bleeding, and are therefore suitably included under the term " prolapse syndrome."

\section{Conditions Necessary for Easy Performance of Vaginal Operation}

1. The uterus and appendages must be mobile.

2. The uterus must not be too large or associated with large tubal or ovarian swellings.

3. The uterine disease must be such as not to contraindicate its removal from below-that is, carcinoma of the cervix.

4. The cervix should come down readily to the vulva.

5. The vaginal outlet should be relaxed, or at least easily enlarged by a relatively sma!l incision, something considerably short of the old Schuchardt's* incision.

Quite often these patients have the fat, more or less pendulous, abdomen, already referred to as unsuitable for abdominal incision.

TECHNIQUE OF THE OPERATION

Five operative procedures are combined: (1) anterior colporrhaphy, (2) vaginal hysterectomy, (3) reconstruction of the hernia of the pouch of Douglas, (4) posterior colporrhaphy, (5) perineorrhaphy.

I will briefly describe a standard method of linking these procedures.

1. Preparation of the field: the perineum is incised back to the anal margin. The nymphae are sutured to the thighs and traction sutures are inserted in the cervix. Determine area of vaginal mucosa to be removed: $(a)$ of the anterior mucosa, inserting landmark suture to mark the upper end of the new anterior vaginal wall; and $(b)$ of the postericr vaginal mucosa, inserting landmark sutures at the site of the upper end of the new posterior vaginal wall.

2. Removal of redundant tissue ; the anterior and posterior mucosa and the uterus in one technique.

3. Reconstruction of the pelvic diaphragm. Fix the uterine stumps and ovario-pelvic stumps on either side to the upper margin of the new lateral vaginal walls. Join the cut edge of the peritoneum of the utero-vesical pouch to the anterior landmark sutures, thus forming the upper end of the new anterior vaginal wall.

4. Reconstruction of the anterior vaginal wall

5. Remove the redundant peritoneum of the hernia of the pouch of Douglas and suture the peritoneal stumps to the

* Schuchardt's incision: An incision starting in the left vault of the vagina and passing deeply on the left side of the anus through the levator ani to the sacrum. posterior landmark sutures, thus forming the upper end of the new posterior vaginal wall.

6. Reconstruct posterior vaginal mucosa and the perineal body.

The operation in my hands requires an hour to an hour and a quarter for its performance, without undue difficulty in the control of unexpected oozing. There is, as a rule, no appreciable shock, and the patients do not have a stormy post-operative period. In general they are sitting up the next day, ready to do justice to-their dinner. Towards the end of a week there is usually some vaginal discharge, which clears up readily with douching. The length of stay in hospital and the after convalescence period is the same as that for simple repair operations.

\section{ADVANTAGES OF THE METHOD}

1. The avoidance of two operations and the prolonged period of convalescence associated therewith, approx:mately five weeks instead of ten.

2. The avoidance of an abdominal incision in fat women and the possible post-operative complications.

3. The relative absence of post-operative shock.

I have looked up the notes of sixty cases. In all, the anatomical resu.t* at the time of departure from hospital was good and the patients' general condition satisfactory. As far as the evidence goes at the moment this procedure would seem to be an improvement on the older method of two operations at different times by different routes.

Forty-four patients have been examined twelve months or more after operation, and fifteen three months or more. The anatomical result* was good, but in one case the posterior fornix, owing to imperfect fixation, bu'ged down to the outlet on straining, as a hernial protrusion. This gave rise to some discomfort, and has been repaired with success. Sixteen out of the sixty cases had post-operative $B$. coli, cystitis, but in eight of these it was known to be lc.ng-standing. There were four slight secondary haemorrhages: one on the seventh day, and three between the tenth and twelfth days. Two patients had small abscesses in the perineum, which cleared up.

One case proved fatal. This happened in a mentally deficient woman who, by means of her fingers, removed the perineal stitches, became septic, and ultimately died from a pulmonary embolism. It is unfortunate that we were unable to recognize this woman's mental condition previous to the operation, for failure to do so has impaired what might otherwise have been an unblemished record.

* Good anatomical result.-The new vagina and perineum approximate to the nulliparous condition, the vagina admitting two fingers with some discomfort and No. 5 vaginal dilator without discomfort. There is no pouting of vaginal walls on straining, and no incontinence of urine on coughing.

With the object of promoting biochemical studies and research a Biochemical Society has been formed in Calcutta, and was formally inaugurated on July 6th at the All-India Institute of Hygiene. The first committee of the society has been composed of the following: Professor N. M. Basu, Lieut.-Colonel T. C. Boyd, Professor S. Ghosh, Professor J. N. Mukherjee, Dr. B. B. Sen, Professor H. K. Sen, Professor H. E. C. Wilson, with Dr. B. C. Guha as honorary secretary and Dr. B. Ahmad as honorary treasurer. It has been arranged to hold monthly meetings for biochemical discussions and reading of original papers. Four meetings have already been held, in which the following papers have been read: B. Ahmad, "The Metabolism of Carotene " ; A. C. Roy, "A Purely Vegetable Medium for the Cultivation of Micro-organisms". S. L. Banerjee and H. K. Sen, "The Catalytic Activation of Diastase" ; A. R. Ghosh and B. C. Guha, "Vitamin C in Indian Foodstuffs " ; R. C. Bhattacharjee, "Snake Venom and its Therapeutic Applications." 